\title{
AUTOMATIC CONTINUITY FOR THE UNITARY GROUP
}

\author{
TODOR TSANKOV
}

(Communicated by Thomas Schlumprecht)

\begin{abstract}
We show that every homomorphism from the infinite-dimensional unitary or orthogonal group to a separable group is continuous.
\end{abstract}

\section{INTRODUCTION}

Many uncountable groups come naturally equipped with a group topology that greatly facilitates their study. When dealing with topological groups, one can use powerful tools such as Haar measure, Lie group theory, and Baire category methods. On the other hand, if one wants to study the groups abstractly, far fewer tools are available. One way to approach this problem is via reconstruction theorems that recover the topology of the group from its algebraic structure. Perhaps the strongest results of this type in the literature have the following form: let $G$ be some Polish group; then every homomorphism from $G$ to a separable group is continuous. (One always needs some restriction on the target group to obtain this type of conclusion in order to avoid the trivial example of the identity map from $G$ to itself equipped with the discrete topology. Separability is a rather mild and natural restriction in this setting.) If $G$ is such a group, we will say that it has the automatic continuity property.

The first automatic continuity results of this type were obtained by Kechris and Rosendal 9], using the techniques of ample generics developed by Hodges, Hodkinson, Lascar, and Shelah 44. Soon thereafter other results in the same vein followed: Rosendal and Solecki [16], Rosendal [13, Kittrell and Tsankov [10; see the recent survey [14] for more details.

The following is the main theorem of this paper. It answers a question of Rosendal [13.

Theorem 1. The unitary (respectively, orthogonal) group of an infinite-dimensional, separable, complex (respectively, real) Hilbert space, equipped with the strong operator topology, has the automatic continuity property.

The proof of this theorem relies in an essential way on recent work of Ben Yaacov, Berenstein and Melleray [2], who, extending the approach of [9], developed a new theory of topometric groups with ample generics that we will briefly describe. They observed that Polish groups that are naturally represented as isometry groups of metric spaces, apart from the Polish topology of pointwise convergence, also

Received by the editors September 6, 2011 and, in revised form, December 23, 2011.

2010 Mathematics Subject Classification. Primary 54H12.

Key words and phrases. Unitary group, automatic continuity.

This research was partially supported by the ANR network AGORA, NT09-461407.

(C) 2013 American Mathematical Society 
carry a natural bi-invariant uniform structure, namely, the one induced by uniform convergence on the metric space. For example, for the unitary group of an infinite-dimensional Hilbert space, the Polish topology is the strong operator topology and the bi-invariant uniform structure is given by the operator norm. One of the main results of 2 is that if such a group $G$ has ample topometric generics, then any homomorphism from $G$ to a separable group that is continuous in the uniform topology is also continuous in the Polish topology. This reduces the task of proving the automatic continuity property for such a Polish group $G$ to proving it for $G$ equipped with the uniform topology, which is a priori easier. They also found examples of groups with ample topometric generics; the ones that will be important for us are the unitary group and the automorphism group of a Lebesgue probability space $\operatorname{Aut}(X, \mu)$. Using this theorem and previous work of Kittrell and Tsankov [10], they were able to show that $\operatorname{Aut}(X, \mu)$ has the automatic continuity property.

To avoid repetition, in the remarks below we concentrate on the unitary group, but they are also valid for the orthogonal group.

One immediate corollary of our theorem is that every action of the unitary group $U(H)$ by homeomorphisms on a compact metrizable space or by isometries on a separable metric space is automatically continuous. Thus, by Gromov and Milman [3], every action of $U(H)$ on a compact metrizable space has a fixed point, and Kirillov's and Olshanski's classification [8,11 of continuous unitary representations of $U(H)$ becomes a classification of all representations of the discrete group $U(H)$ on a separable Hilbert space, etc.

Combining Theorem [1] with the result of Stojanov [17 that the unitary group is totally minimal (i.e. every continuous homomorphism to a Hausdorff topological group is open), we obtain the following corollary.

Corollary 2. Let $G$ be the unitary or the orthogonal group. Then the following hold:

(i) $G$ admits a unique separable group topology;

(ii) if $G^{\prime}$ is a Polish group and $\phi: G \rightarrow G^{\prime}$ a homomorphism, then $\phi(G)$ is a closed subgroup of $G^{\prime}$.

Atim 1 had previously shown that the unitary and orthogonal groups admit a unique Polish group topology.

This corollary rules out the existence of non-trivial homomorphisms from $U(H)$ to Polish locally compact groups or, more generally, Polish groups admitting a left-invariant complete metric or Polish totally disconnected groups.

Another corollary of the theorem is that the quotient of the unitary group by the normal subgroup of unitary operators that differ from the identity by a compact operator does not admit a non-trivial homomorphism to a separable group. This generalizes a result of Pickrell [12, who had shown that this group does not admit continuous (with respect to the quotient of the norm topology) non-trivial unitary representations on a separable Hilbert space.

Theorem 1 should be contrasted with the situation for finite-dimensional unitary groups: it is a result of Kallman [6], and independently of Thomas [18, that $\mathrm{GL}(n, \mathbf{C})$ embeds as a subgroup of $S_{\infty}$ and, as $U(n)$ is connected, the restriction of this embedding to $U(n)$ cannot be continuous. 
Remark. In [13], Rosendal asks whether there is an (infinite) compact metrizable group that satisfies the automatic continuity property. It follows from the PeterWeyl theorem and the result mentioned above that every compact metrizable group embeds in $S_{\infty}$, and therefore if such a group satisfies the automatic continuity property, it must be profinite.

\section{Proofs}

We set to prove Theorem 1 and first concentrate on the complex case. Let $H$ be an infinite-dimensional, separable, complex Hilbert space and $U(H)$ its unitary group.

A subset $W$ of a group is called symmetric if $W=W^{-1}$. A symmetric set is called countably syndetic if countably many left (or, equivalently, right) translates of it cover the group. By [16, Proposition 2], to establish Theorem 11, it suffices to prove the following.

Theorem 3. If $W$ is a symmetric, countably syndetic subset of $U(H)$, then $W^{506}$ contains an open (in the strong operator topology) neighborhood of the identity.

The constant 506 above has no special significance and is an artifact of the proof; any fixed constant would do for our purposes.

By the results of [2], to prove Theorem 3], it suffices to find a subset of $W^{496}$ that is open in the norm topology, and this is what we do below. As we are going to use automatic continuity results for various subgroups of $U(H)$, we start with the following preliminary lemma.

Lemma 4. Suppose that $G$ is a group, $G^{\prime}$ is a subgroup and $W$ is a countably syndetic set for $G$. Then $G^{\prime} \cap W^{2}$ is countably syndetic for $G^{\prime}$.

Proof. Let $G=\bigcup_{n} g_{n} W$ and $A=\left\{n \in \mathbf{N}: g_{n} W \cap G^{\prime} \neq \emptyset\right\}$. For every $n \in A$, choose $h_{n} \in g_{n} W \cap G^{\prime}$ so that $h_{n}=g_{n} w_{n}$ with $w_{n} \in W$. Now let $h \in G^{\prime}$ be arbitrary. There exists $n \in A$ and $w \in W$ such that $h=g_{n} w$. Then $h=h_{n} w_{n}^{-1} w \in h_{n}\left(W^{2} \cap G^{\prime}\right)$, showing that $G^{\prime}=\bigcup_{n \in A} h_{n}\left(W^{2} \cap G^{\prime}\right)$.

We establish some notation. If $K$ is a closed subspace of $H$, denote by $U(K)$ the unitary group of $K$ and by $G_{K}$ the pointwise stabilizer of $K$ in $U(H)$ :

$$
G_{K}=\{u \in U(H): u x=x \text { for all } x \in K\}
$$

so that $U(K)$ is naturally isomorphic to $G_{K^{\perp}}$. Denote by $I_{K}$ the setwise stabilizer of $K$ in $U(H)$ and note that $I_{K}=G_{K} \times G_{K^{\perp}}$. Define $P_{K}: I_{K} \rightarrow G_{K^{\perp}}$ as follows: if $u=u_{1} u_{2} \in I_{K}$ with $u_{1} \in G_{K}$ and $u_{2} \in G_{K^{\perp}}$, let $P_{K} u=u_{2}$. If $K$ is a subspace of $H$, say that $K$ is balanced if both $K$ and $K^{\perp}$ are infinite-dimensional.

Now fix a countably syndetic $W \subseteq U(H)$. Then there exists a countable subset $\left\{s_{n}: n \in \mathbf{N}\right\} \subseteq U(H)$ such that $U(H)=\bigcup_{n} s_{n} W$.

Say that a set $A \subseteq U(H)$ is full for a subspace $K \subseteq H$ if for every $u \in U(K)$, there exists $v \in A \cap I_{K}$ such that $\left.v\right|_{K}=u$. The following diagonalisation argument has by now become quite standard.

Lemma 5. Suppose that $H=\bigoplus_{n} K_{n}$, where each $K_{n}$ is infinite-dimensional. Then there exists $n$ such that $W^{2}$ is full for $K_{n}$.

Proof. It suffices to see that some $s_{n} W$ is full for $K_{n}$ because then

$$
W^{2}=\left(s_{n} W\right)^{-1}\left(s_{n} W\right)
$$


would be full as well. If not, then for each $n$, there exists $u_{n} \in U\left(K_{n}\right)$ such that for all $u \in s_{n} W$ that leave $K_{n}$ invariant, $\left.u\right|_{K_{n}} \neq u_{n}$. Then $\bigoplus_{k} u_{k} \in U(H)$, but $\bigoplus_{k} u_{k} \notin s_{n} W$ for all $n$, a contradiction.

The proof of the following lemma is similar to the one of [10, Lemma 3.3].

Lemma 6. Suppose that $H=\bigoplus_{n} K_{n}$, where each $K_{n}$ is infinite-dimensional. Then there exists $n$ such that $G_{K_{n}^{\perp}} \subseteq W^{24}$.

Proof. A unitary operator $u \in U(H)$ is called a symmetry if $u^{2}=1$; i.e. there exists a decomposition $H=H_{1} \oplus H_{2}$ such that $u x=x$ for all $x \in H_{1}$ and $u x=-x$ for all $x \in H_{2}$. Note that every two symmetries for which the corresponding eigenspaces $H_{1}$ and $H_{2}$ are infinite-dimensional are conjugate in $U(H)$. Halmos and Kakutani [5] have shown that every unitary operator is the product of four symmetries. Even though they do not mention it explicitly, it follows from their proof that one can choose the symmetries so that they have infinite-dimensional eigenspaces.

Now let $K_{n}$ be such that $W^{2}$ is full for $K_{n}$ as given by Lemma 5 . Let $e_{1}, e_{2}, \ldots$ be an orthonormal basis of $K_{n}$ and let $\left\{A_{i}: i \in 2^{\aleph_{0}}\right\}$ be a family of subsets of $\mathbf{N}$ such that $A_{i_{1}} \triangle A_{i_{2}}$ is infinite and co-infinite if $i_{1} \neq i_{2}$. Let $v_{i} \in U\left(K_{n}\right)$ be the symmetry defined by

$$
v_{i} e_{j}= \begin{cases}e_{j}, & \text { if } j \in A_{i} ; \\ -e_{j}, & \text { if } j \notin A_{i},\end{cases}
$$

and let $u_{i}=v_{i} \oplus 1_{K_{n}^{\perp}} \in U(H)$. By the pigeonhole principle, there exist $i_{1} \neq i_{2}$ and $n$ such that $u_{i_{1}}, u_{i_{2}} \in s_{n} W$. Then $u_{i_{1}} u_{i_{2}}=u_{i_{1}}^{-1} u_{i_{2}} \in W^{2}$, and by the choice of the sets $A_{i}, v_{i_{1}} v_{i_{2}}$ is a symmetry of $K_{n}$ with infinite-dimensional eigenspaces. Applying the result of [5], we obtain that every element of $U\left(K_{n}\right)$ is a product of four conjugates of $v_{i_{1}}^{-1} v_{i_{2}}$. Finally, by the fullness of $W^{2}$ for $K_{n}$ and the fact that conjugation of elements of $G_{K_{n}^{\perp}}$ by elements of $I_{K_{n}}$ cancels out on $K_{n}^{\perp}$, we have that $G_{K_{n}^{\perp}} \subseteq\left(W^{2} W^{2} W^{2}\right)^{4}=W^{24}$.

Let $(X, \mu)$ be a standard probability space (isomorphic to the interval $[0,1]$ with Lebesgue measure) and denote by $\operatorname{Aut}(X, \mu)$ the group of all measure-preserving automorphisms of $(X, \mu)$. We equip this group with the weak topology, which is the coarsest topology that makes the maps $\operatorname{Aut}(X, \mu) \rightarrow \mathbf{R}, T \mapsto \mu(T(A) \triangle B)$, for $A$ and $B$ measurable subsets of $X$, continuous. We also consider the uniform distance $d$ on $\operatorname{Aut}(X, \mu)$ defined by

$$
d\left(T_{1}, T_{2}\right)=\mu\left(\left\{x \in X: T_{1} x \neq T_{2} x\right\}\right) .
$$

Note that the topology defined by $d$ is strictly finer than the weak topology.

Lemma 7. Let $V \subseteq \operatorname{Aut}(X, \mu)$ be a countably syndetic subset of $\operatorname{Aut}(X, \mu)$. Then $V^{48}$ contains a weak neighborhood of the identity.

Proof. This is a combination of results of [10] and [2]. By [10, Theorem 3.1], $V^{38}$ contains a $d$-ball $B_{\epsilon}$ of radius $\epsilon$ around the identity in $\operatorname{Aut}(X, \mu)$ for some $\epsilon>0$. (Even though the paper [10] deals with full groups of equivalence relations, this proof works equally well for the entire group $\operatorname{Aut}(X, \mu)$.) Applying the fact that $\operatorname{Aut}(X, \mu)$ has ample topometric generics (see [2, Section 5.4]) and [2, Theorem 4.4], we obtain that $V^{38} V^{10}=V^{48}$ contains a weak neighborhood of the identity. 
We now return to the unitary group.

Lemma 8. There exists a balanced subspace $K$ of $H$ such that $G_{K} \subseteq W^{24}$ and $W^{120}$ contains an open neighborhood of the identity in $I_{K}$.

Proof. Let $H=\bigoplus_{n} K_{n}$ be a decomposition of $H$ into infinite-dimensional subspaces and let $K_{n}$ be the subspace given by Lemma 6. Put $K=K_{n}^{\perp}$ so that $G_{K} \subseteq W^{24}$.

Consider the probability space $(X, \mu)=\left(\mathbf{R}^{\mathbf{N}}, \nu^{\mathbf{N}}\right)$, where $\nu$ denotes the standard Gaussian measure. Then there exists an embedding $\gamma: U(K) \rightarrow \operatorname{Aut}(X, \mu)$ which has the following property: if $\kappa: \operatorname{Aut}(X, \mu) \rightarrow U\left(L^{2}(X, \mu)\right)$ denotes the standard embedding, then there exists a balanced subspace $K^{\prime} \subseteq L^{2}(X, \mu)$ and a unitary isomorphism $\Phi: K \rightarrow K^{\prime}$ such that

$$
\left.(\kappa \circ \gamma)(u)\right|_{K^{\prime}}=\Phi u \Phi^{-1} \quad \text { for every } u \in U(K) .
$$

This is the so-called Gaussian construction; see, for example, [7, Appendix E] for details.

Identify $H$ with $L^{2}(X, \mu)$ via an isomorphism which sends $K$ to $K^{\prime}$ and whose restriction to $K$ is equal to $\Phi$. We thus obtain embeddings

$$
U(K) \stackrel{\gamma}{\rightarrow} \operatorname{Aut}(X, \mu) \stackrel{\kappa}{\rightarrow} U(H)
$$

such that if we put $\theta=\kappa \circ \gamma$, then $K$ is invariant under $\theta(U(K))$, and for every $u \in U(K),\left.\theta(u)\right|_{K}=u$.

Equip the three groups $U(K)$, Aut $(X, \mu)$ and $U(H)$ with their Polish topologies and observe that both $\gamma$ and $\kappa$ are homeomorphic embeddings. By Lemma 4, $W^{2} \cap \kappa(\operatorname{Aut}(X, \mu))$ is countably syndetic in $\kappa(\operatorname{Aut}(X, \mu))$. By Lemma 7 ,

$$
\left(W^{2} \cap \kappa(\operatorname{Aut}(X, \mu))\right)^{48} \subseteq W^{96}
$$

contains an open neighborhood of the identity in $\kappa(\operatorname{Aut}(X, \mu))$, and, in particular, there exists an open neighborhood of the identity $O$ in $\theta(U(K))$ such that $O \subseteq W^{96}$.

Let $\tau$ be the natural isomorphism $U(K) \rightarrow G_{K^{\perp}}$ given by $\tau(v)=v \oplus 1_{K^{\perp}}$ and let $\rho=\theta \circ \tau^{-1}$ so that $\rho$ is an isomorphism $G_{K^{\perp}} \rightarrow \theta(U(K))$. We also note that for every $u \in G_{K^{\perp}}, P_{K}(\rho(u))=u$. Let $O^{\prime}=\rho^{-1}(O) \subseteq G_{K^{\perp}}$. We now check that $O^{\prime} G_{K} \subseteq W^{120}$, and as $O^{\prime} G_{K}$ is open in $G_{K^{\perp}} \times G_{K}=I_{K}$, this will complete the proof. Let $\left(u_{1}, u_{2}\right) \in O^{\prime} \times G_{K}$. We have

$$
\begin{aligned}
u_{1} u_{2} & =u_{1} P_{K^{\perp}}\left(\rho\left(u_{1}\right)\right) P_{K^{\perp}}\left(\rho\left(u_{1}\right)\right)^{-1} u_{2} \\
& =P_{K}\left(\rho\left(u_{1}\right)\right) P_{K^{\perp}}\left(\rho\left(u_{1}\right)\right) P_{K^{\perp}}\left(\rho\left(u_{1}\right)\right)^{-1} u_{2} \\
& =\rho\left(u_{1}\right)\left(P_{K^{\perp}}\left(\rho\left(u_{1}\right)\right)^{-1} u_{2}\right) \in O G_{K} \subseteq W^{96} W^{24}=W^{120} .
\end{aligned}
$$

Lemma 9. Let $K$ and $L$ be two infinite-dimensional subspaces of a Hilbert space $H$. Then there exist two infinite-dimensional subspaces $K^{\prime} \subseteq K$ and $L^{\prime} \subseteq L$ such that $K^{\prime} \perp L^{\prime}$.

Proof. We inductively build two orthonormal sequences, $e_{1}, e_{2}, \ldots \in K$ and $f_{1}, f_{2}$, $\ldots \in L$, such that $\left\langle e_{i}, f_{j}\right\rangle=0$ for all $i, j$. First pick a unit vector $e_{1} \in K$ arbitrarily. Assuming that $e_{1}, f_{1}, e_{2}, f_{2}, \ldots, e_{n}$ have been constructed, choose $f_{n} \in L$ and $e_{n+1} \in K$ so that $f_{n}$ is perpendicular to $e_{1}, f_{1}, \ldots, e_{n}$ and $e_{n+1}$ is perpendicular to $e_{1}, f_{1}, \ldots, e_{n}, f_{n}$ (this can be done because both $K$ and $L$ are infinite-dimensional). Finally, let $K^{\prime}=\operatorname{span}\left\{e_{i}\right\}, L^{\prime}=\operatorname{span}\left\{f_{i}\right\}$. 
For the next lemma, recall that $S_{\infty}$ is the Polish group of all permutations of an infinite, countable, discrete set, equipped with the pointwise convergence topology.

Lemma 10. Let $K$ be a subspace of $H$ that satisfies the conclusion of Lemma 8 and let $L \subseteq H$ be infinite-dimensional. Then there exists $v \in W^{64}$ and $L^{\prime} \subseteq L$ such that $v L^{\prime}=K^{\perp}$.

Proof. By Lemma 9] there exist infinite-dimensional $K_{1} \subseteq K^{\perp}$ and $L_{1} \subseteq L$ such that $K_{1} \perp L_{1}$. Let $B_{1}$ and $B_{1}^{\prime}$ be orthonormal bases of $K_{1}$ and $L_{1}$ respectively and consider the embedding $\theta_{1}: S_{\infty} \rightarrow G_{\left(K_{1} \oplus L_{1}\right)^{\perp}}$ given by the action of $S_{\infty}$ on $K_{1} \oplus L_{1}$ by permuting the basis $B_{1} \cup B_{1}^{\prime}$. Let $Q_{1}=\theta_{1}\left(S_{\infty}\right)$. By Lemma 4, $W^{2} \cap Q_{1}$ is countably syndetic for $Q_{1}$, so by the proof of [9, Theorem 6.24] (see also [16], where this fact is mentioned explicitly), $\left(W^{2} \cap Q_{1}\right)^{10} \subseteq W^{20}$ contains an open subgroup $V_{1}$ of $Q_{1}$. Then there exist an element $v_{1} \in V_{1}$ and infinite-dimensional subspaces $K_{2} \subseteq K_{1}$ and $L_{2} \subseteq L_{1}$ such that $v_{1} L_{2}=K_{2}$.

Now let $B_{2}, B_{2}^{\prime}, B_{2}^{\prime \prime}$ be orthonormal bases of $K_{2}, K^{\perp} \ominus K_{2}$, and $K$, respectively. Let $\theta_{2}: S_{\infty} \rightarrow U(H)$ be the embedding given by the action of $S_{\infty}$ on $H$ by permuting the basis $B_{2} \cup B_{2}^{\prime} \cup B_{2}^{\prime \prime}$ and let $Q_{2}=\theta_{2}\left(S_{\infty}\right)$. Then, as above, $W^{20}$ contains an open subgroup $V_{2}$ of $Q_{2}$. A basic open subgroup of $Q_{2}$ is the stabilizer of finitely many elements of the basis $B_{2} \cup B_{2}^{\prime} \cup B_{2}^{\prime \prime}$. Suppose that $V_{2}$ is the stabilizer (in $Q_{2}$ ) of $A \cup A^{\prime} \cup A^{\prime \prime}$, where $A, A^{\prime}, A^{\prime \prime}$ are finite subsets of $B_{2}, B_{2}^{\prime}, B_{2}^{\prime \prime}$, respectively. As $B_{2}$ is infinite, there exists $v_{2} \in G_{K} \cap Q_{2}$ such that $v_{2}\left(B_{2}\right) \supseteq A \cup A^{\prime}$. Finally, let $v_{3} \in V_{2}$ be such that $v_{3}\left(v_{2}\left(B_{2}\right)\right)=B_{2} \cup B_{2}^{\prime}$ so that $v_{3}\left(v_{2}\left(K_{2}\right)\right)=K^{\perp}$. (We can achieve this as follows: let $C_{1}^{\prime \prime} \sqcup C_{2}^{\prime \prime}$ be any splitting of $B_{2}^{\prime \prime} \backslash A^{\prime \prime}$ into two infinite pieces; then define $v_{3}$ to be a permutation of $B_{2}^{\prime \prime} \cup B_{2} \cup B_{2}^{\prime}$ that fixes $A \cup A^{\prime} \cup A^{\prime \prime}$ and sends $B_{2}^{\prime \prime} \backslash A^{\prime \prime}$ to $C_{1}^{\prime \prime}, v_{2}\left(B_{2}\right) \backslash\left(A \cup A^{\prime}\right)$ to $\left(B_{2} \cup B_{2}^{\prime}\right) \backslash\left(A \cup A^{\prime}\right)$, and $\left(B_{2} \cup B_{2}^{\prime}\right) \backslash v_{2}\left(B_{2}\right)$ to $C_{2}^{\prime \prime}$.)

We finally have that $v_{3} v_{2} v_{1} L_{2}=K^{\perp}$ and

$$
v_{3} v_{2} v_{1} \in V_{2} G_{K} V_{1} \subseteq W^{20} W^{24} W^{20}=W^{64},
$$

completing the proof of the lemma.

If $r>0$, denote by $B_{r}$ the open ball of radius $r$ around the identity in $U(H)$ in the operator norm.

Lemma 11. There exists $\epsilon>0$ such that $B_{\epsilon} \subseteq W^{496}$.

Proof. Suppose $K \subseteq H$ is as in the conclusion of Lemma 8 and let $\epsilon>0$ be such that $B_{\epsilon} \cap I_{K} \subseteq W^{120}$. We will now check that $B_{\epsilon} \subseteq W^{496}$. Let $u \in B_{\epsilon}$ and let $L$ be a balanced subspace of $H$ invariant under $u$ (which exists by the spectral theorem). Let $u_{1}=P_{L} u$ and $u_{2}=P_{L^{\perp}} u$ so that $u=u_{1} u_{2}$ and note that $u_{1}, u_{2} \in B_{\epsilon}$. By Lemma 10, there exists $L^{\prime} \subseteq L^{\perp}$ and $v_{1} \in W^{64}$ such that $v_{1} L^{\prime}=K^{\perp}$; in particular, $v_{1} u_{1} v_{1}^{-1} \in G_{K^{\perp}} \subseteq I_{K}$. As the norm is invariant under conjugation, we have that $v_{1} u_{1} v_{1}^{-1} \in B_{\epsilon} \cap I_{K} \subseteq W^{120}$, whence $u_{1} \in W^{64} W^{120} W^{64}=W^{248}$. A similar argument shows that $u_{2} \in W^{248}$, so $u \in W^{496}$ and we are done.

Now it is easy to complete the proof of Theorem 3. If $A \subseteq U(H)$, denote

$$
(A)_{\epsilon}=\{u \in U(H): \exists a \in A\|a-u\|<\epsilon\} .
$$

Let $\epsilon$ be the one given by Lemma 11, By [2, Theorem 4.4], $\left(W^{10}\right)_{\epsilon}$ contains an open (in the strong operator topology) neighborhood of the identity, but $\left(W^{10}\right)_{\epsilon}=$ $W^{10} B_{\epsilon} \subseteq W^{506}$. This concludes the proof of Theorem 3 in the complex case. 
We now indicate the necessary changes in the proof to establish the theorem in the case of a real Hilbert space $H$. As the proof contains practically no analytic arguments, these changes are rather minor. We replace the occasional use of the spectral theorem by the following standard lemma.

Lemma 12. Let $H$ be a real, infinite-dimensional Hilbert space and $T$ an orthogonal operator. Then there exists a decomposition $H=\bigoplus_{n \in \mathbf{N}} H_{n}$ such that each $H_{n}$ is infinite-dimensional and invariant under $T$.

Proof. Let $H_{\mathbf{C}}=H \otimes \mathbf{C}$ be the complexification of $H$ and let $T^{\mathbf{C}}$ be the complexification of $T$ so that $T^{\mathbf{C}} \in U\left(H_{\mathbf{C}}\right)$. There is a natural operation of conjugation on $H_{\mathbf{C}}, \xi \otimes z \mapsto \xi \otimes \bar{z}$, one can identify $H$ with the real subspace of $H^{\mathbf{C}}$ given by $\left\{\eta \in H^{\mathbf{C}}: \eta=\bar{\eta}\right\}, T^{\mathbf{C}}$ commutes with conjugation, and $\left.T^{\mathbf{C}}\right|_{H}=T$. By the spectral theorem, $H^{\mathbf{C}}$ decomposes as a sum $\bigoplus_{n} K_{n}$ of $T^{\mathbf{C}}$-invariant infinite-dimensional subspaces, and by rearranging, we can further assume that $K_{n}=\overline{K_{n}}$ for each $n$. Then for each $n, H_{n}=\left\{\eta+\bar{\eta}: \eta \in K_{n}\right\}$ is an infinite-dimensional subspace of $H$, and the $H_{n}$ s are pairwise orthogonal and invariant under $T$.

We go through the lemmas above one by one. The proof of Lemma 5 goes through verbatim in the real case. The proof of Lemma 6 goes through verbatim as well except that in the proof of the Halmos-Kakutani theorem we need to replace the use of the spectral theorem by Lemma[12, Lemmas 8, 9, and 10] survive without changes (we note that the Gaussian construction works equally well and, in a sense, even more easily in the real case). In Lemma 11, we need once again to invoke Lemma 12 , Finally, to complete the proof, in order to apply [2, Theorem 4.4], we need to check that the orthogonal group has ample topometric generics. To verify this for the unitary group, the authors of [2] use a result of Rosendal ([15, Proposition 6.6]). As the author of [15] notes explicitly, his proof works equally well for the orthogonal group.

Proof of Corollary 2. (1i) Let $\tau$ denote the natural Polish topology on $G$ and let $\tau^{\prime}$ be some other separable topology. Then the identity map $(G, \tau) \rightarrow\left(G, \tau^{\prime}\right)$ is continuous by Theorem 1 and open by the theorem of Stojanov [17] and is therefore a homeomorphism.

(iii) If $G^{\prime}$ is Polish and $\phi: G \rightarrow G^{\prime}$ is a homomorphism, then $\phi$ is continuous by Theorem 1 and open by [17]. Then $\phi(G)$ is isomorphic (as a topological group) with $G / \operatorname{ker} \phi$, therefore Polish, whence closed in $G^{\prime}$.

\section{ACKNOWLEDGEMEnTS}

Part of this work was carried out during a visit of the author to Caltech. He is grateful to Alexander Kechris and the Caltech mathematics department for their hospitality. The author is also grateful to Julien Melleray for some useful discussions on the topic of the paper and for pointing out an error in a preliminary draft, to Christian Rosendal for supplying a reference, and to the anonymous referee for some helpful comments.

\section{ReFERENCES}

[1] Alexandru Atim, Uniqueness results for the infinite unitary, orthogonal and associated groups, Ph.D. Thesis, University of North Texas, 2008. MR.2712123

[2] Itaï Ben Yaacov, Alexander Berenstein, and Julien Melleray, Polish topometric groups, 2010. preprint available at arXiv:1007.3367. 
[3] M. Gromov and V. D. Milman, A topological application of the isoperimetric inequality, Amer. J. Math. 105 (1983), no. 4, 843-854. MR708367 (84k:28012)

[4] Wilfrid Hodges, Ian Hodkinson, Daniel Lascar, and Saharon Shelah, The small index property for $\omega$-stable $\omega$-categorical structures and for the random graph, J. London Math. Soc. (2) 48 (1993), no. 2, 204-218. MR.1231710 (94d:03063)

[5] Paul R. Halmos and Shizuo Kakutani, Products of symmetries, Bull. Amer. Math. Soc. 64 (1958), 77-78. MR0100225 (20:6658)

[6] Robert R. Kallman, Every reasonably sized matrix group is a subgroup of $S_{\infty}$, Fund. Math. 164 (2000), no. 1, 35-40. MR.1784652 (2001h:20005)

[7] Alexander S. Kechris, Global aspects of ergodic group actions, Mathematical Surveys and Monographs, vol. 160, American Mathematical Society, Providence, RI, 2010. MR2583950 (2011b:37003)

[8] A. A. Kirillov, Representations of the infinite-dimensional unitary group, Dokl. Akad. Nauk. SSSR 212 (1973), 288-290. MR0340487(49:5239)

[9] Alexander S. Kechris and Christian Rosendal, Turbulence, amalgamation and generic automorphisms of homogeneous structures, Proc. Lond. Math. Soc. 94 (2007), no. 2, 302-350. MR2308230 (2008a:03079)

[10] John Kittrell and Todor Tsankov, Topological properties of full groups, Ergodic Theory Dynam. Systems 30 (2010), no. 2, 525-545. MR2599891 (2011d:37006)

[11] G. I. Olshanski, Unitary representations of the infinite-dimensional classical groups $\mathrm{U}(p, \infty)$, $\mathrm{SO}_{0}(p, \infty), \operatorname{Sp}(p, \infty)$, and of the corresponding motion groups, Funktsional. Anal. i Prilozhen. 12 (1978), no. 3, 32-44, 96.

[12] Doug Pickrell, The separable representations of $\mathrm{U}(H)$, Proc. Amer. Math. Soc. 102 (1988), no. 2, 416-420. MR921009 (89c:22036)

[13] Christian Rosendal, Automatic continuity in homeomorphism groups of compact 2-manifolds, Israel J. Math. 166 (2008), 349-367. MR2430439 (2009f:57055)

[14] _ Automatic continuity of group homomorphisms, Bull. Symbolic Logic 15 (2009), no. 2, 184-214. MR2535429 (2011e:03064)

[15] _ A topological version of the Bergman property, Forum Math. 21 (2009), no. 2, 299332. MR2503307 (2010a:03054)

[16] Christian Rosendal and Sławomir Solecki, Automatic continuity of homomorphisms and fixed points on metric compacta, Israel J. Math. 162 (2007), 349-371. MR2365867 (2009d:54016)

[17] L. Stojanov, Total minimality of the unitary groups, Math. Z. 187 (1984), no. 2, 273-283. MR753438 (85k:22008)

[18] Simon Thomas, Infinite products of finite simple groups. II, J. Group Theory 2 (1999), no. 4, 401-434. MR 1718758(2000m:20043)

Institut de Mathématiques de Jussieu, CAse 7012, Université Paris 7, 75205 Paris Cedex 13, France

E-mail address: todor@math.jussieu.fr 